\title{
Ägyptologie und Koptologie in Göttingen. Zur Geschichte einer (nicht immer) wunderbaren Freundschaft
}

Ziel meines Vortrags ist es, die wechselvolle Beziehung zwischen Ägyptologie und Koptologie, besonders an der Universität Göttingen, von den Anfängen bis in die Gegenwart nachzuzeichnen. Da auch die Fachgrenzen selbst historisch bedingt sind und nicht immer klar gezogen waren, vor$a b$ eine Arbeitsdefinition für diese beiden Fächer: Unter Ägyptologie verstehe ich die Wissenschaft vom pharaonischen Ägypten von der Jungsteinzeit bis in die ersten Jahrhunderte unserer Zeitrechnung. Sie beschäftigt sich mit der Geschichte, den religiösen, sozialen und politischen Verhältnissen eines Landes, das ungefähr dem heutigen Staatsgebiet Ägyptens entspricht, mit der altägyptischen Sprache und den in ihr geschriebe-

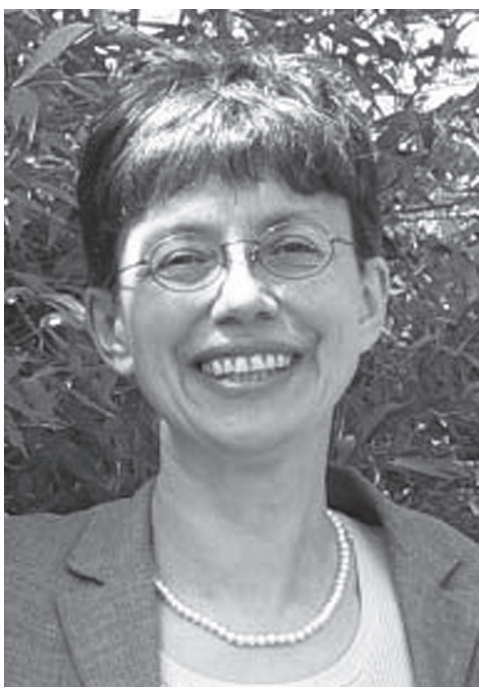

Heike Behlmer, Professorin der Ägyptologie und Koptologie an der Georg-August-Universität Göttingen, O. Mitglied der Göttinger Akademie seit 2012 nen Texten. Koptologie ist die Wissenschaft vom nachpharaonischen Ägypten und dem ägyptischen Christentum. Damit reichen Ausläufer bis in die Moderne, denn das Christentum existiert heute noch als Minderheitsreligion im Land. Beide Fächer überlappen sich vor allem dadurch, daß das Koptische die letzte Stufe der altägyptischen Sprache ist, die jetzt aber mit einer Buchstabenschrift geschrieben wurde.

Ägyptologie als Fach differenziert sich um die Mitte des 19. Jh. aus dem allgemeinen Gebiet der „Altertumswissenschaften“ heraus. In dieser Phase wird die institutionelle Verankerung der Ägyptologie zum einen durch die Gründung von Museen und Museumsabteilungen für ägyptische Artefakte 
belegt, zum anderen, in der Regel zeitlich etwas später, durch die Gründung von Lehrstühlen für Ägyptologie an bedeutenden deutschen und französischen Universitäten ${ }^{1}$. Koptologie ist als akademisches Fach dagegen eine junge Wissenschaft, die sich erst seit dem 2. Weltkrieg an nur wenigen Universitäten weltweit etabliert hat. Als Forschungsgegenstand ist sie jedoch älter als die Ägyptologie, da koptische Handschriften lange gelesen und studiert wurden, bevor 1822 sich mit der Entzifferung der Hieroglyphen durch Jean-François Champollion ein ganz neuer Zugang zu ägyptischen Denkmälern und zur ägyptischen Geschichte eröffnete. In der Tat wurde das schon gut bekannte Koptische instrumental für diese Entzifferung ${ }^{2}$.

Nach Göttingen kam die Ägyptologie 1867 auch durch die Zeitläufte nach einer eher traurigen Vorgeschichte: der Lehrtätigkeit des früh verstorbenen Privatdozenten Maximilian Adolph Uhlemann ${ }^{3}$. Nach der Annektion des Königreichs Hannover durch Preußen wollte das Unterrichtsministerium einen bestimmten Ägyptologen im Land halten: Heinrich Brugsch. Dieser hatte ein Angebot aus Paris erhalten, das von Napoleon III. unterstützt wurde, ein Angebot nicht ohne politische Brisanz, und so wurde für ihn der Uhlemann verwehrte Lehrstuhl eingerichtet. Heinrich Brugsch war ein populärer Lehrer, jedoch oft nach Ägypten beurlaubt. Er ging dann 1877 auf Einladung des ägyptischen Vizekönigs dauerhaft nach Kairo, um eine Schule für einheimische Ägyptologen aufzubauen ${ }^{4}$ - womit er im übrigen seiner Zeit um ein Jahrhundert voraus war.

$1 \quad$ Die Einrichtung eines Lehrstuhls kann dabei der Institutionalisierung eines Faches um eine gewisse Zeit vorausgehen. Das deutlichste Beispiel dafür ist Jean-François Champollions Lehrstuhl für Archäologie am Collège de France (1831). Champollion hinterließ keine Schule, und sein Nachfolger Jean-Antoine Letronne war ein klassischer Philologe. Die Ägyptologie wurde in Paris erst wieder mit der Ernennung Emmanuel de Rougés 1860 eingerichtet. Auch im deutschsprachigen Raum gab es eine größere Lücke zwischen der Einrichtung des ersten Lehrstuhls für Ägyptologie in Preußen (1846 für Richard Lepsius) und mehreren Neueinrichtungen in der 2. Hälfte des 19. Jh., beginnend 1867 mit Göttingen. In der anglophonen Welt wurde ein Ägyptologielehrstuhl erst 1892 in London für den Archäologen W. M. Flinders Petrie begründet; die USA folgten 1905 (für James Breasted in Chicago).

2 Für die Rolle des Koptischen in der Hieroglyphenentzifferung s. Richard B. Parkinson et al., Cracking Codes. The Rosetta Stone and Decipherment, Berkeley/Los Angeles 1999, Kap. 1, bes. S. 31-35.

3 Uhlemann wurde 1850 in Leipzig als Schüler Gustav Seyffarths promoviert, dessen alternative Deutung der Hieroglyphenschrift sich gegenüber der Schule Champollions nicht durchsetzen sollte. Seine dünne Personalakte (Universitätsarchiv Göttingen, Az. 4 V c 80) reicht von der Erteilung der venia legendi 1854 bis zu seinem frühen Tod 1862. Auch sonst ist wenig über ihn bekannt; einige Bemerkungen zu seiner Promotion finden sich in einer Würdigung von Seyffarths Leben und Werk: Elke Blumenthal, Altes Ägypten in Leipzig. Zur Geschichte des Ägyptischen Museums und des Ägyptologischen Instituts an der Universität Leipzig, Leipzig 1981, 3-7.

4 Zu Heinrich Brugsch s. Ursula Köhler „Die Anfänge der deutschen Ägyptologie: Heinrich Brugsch. Eine Einschätzung“, Göttinger Miszellen 12 (1974), 29-41; Erika Endesfel- 
Die Koptologie, wenn auch noch nicht unter diesem Namen, kam zwei Jahre später als Brugsch nach Göttingen, in Gestalt von Paul de Lagarde, der 1869 als Nachfolger Heinrich Ewalds Professor für Orientalische Philologie wurde. Lagarde ist außerhalb seines Fachgebiets wegen seiner zivilisationskritischen und antisemitischen politischen Schriften bekannt, die gerade erst in jüngster Zeit wieder vermehrt Interesse in der Wissenschaft fanden ${ }^{5}$. Sein wissenschaftliches Erbe ist vor allem die Begründung des Projektes der kritischen Edition des griechischen Alten Testamentes, der Septuaginta. Dieses Göttinger Akademieprojekt, das von seinem Schüler Alfred Rahlfs umgesetzt wurde, hat 2008 sein 100jähriges Jubiläum gefeiert. ${ }^{6}$

In meinen eigenen wissenschaftsgeschichtlichen Arbeiten zu Lagarde geht es mir vor allem um seine Rolle in der sich herausbildenden Ägyptologie und seine Editionen koptischer Texte ${ }^{7}$. Lagardes große Liebe galt, was lange wenig bekannt war, den koptischen Texten und der koptischen Sprache, die er lingua pulcherrima et sapientissima „überaus schöne und gelehrte Sprache“ "nannte ${ }^{8}$. Er war Schüler des früh verstorbenen Koptologen Moritz Gotthilf Schwartze, für den 1845 eine außerplanmäßige Professur in Berlin eingerichtet wurde 9 . Nach seiner Promotion gab Lagarde zunächst kopti-

der, „Schon mit sechzehn hatte er das Demotische entziffert. Der Ägyptologe Heinrich Brugsch (1827-1894)“, Berlinische Monatsschrift, Heft 9, 1998, 58-64 (http://www.luiseberlin.de/bms/bmstext/9809pora.htm; zuletzt besucht am 30.06.2012); Heike Behlmer, „Heinrich Friedrich Karl Brugsch (1827-1894), Ägyptologe. Rede anläßlich der Enthüllung einer Gedenktafel am 26.09.2003, Untere Masch 16“, Göttinger Jahrbuch 51 (2003), 165-169.

5 Ulrich Sieg, Deutschlands Prophet. Paul de Lagarde und die Ursprünge des modernen Antisemitismus, München 2007; ders., „Paul de Lagarde und die völkische Bewegung“, in: Friedrich Wilhelm Graf (Hg.), Intellektuellen-Götter: Das religiöse Laboratorium der klassischen Moderne. Schriften des Historischen Kollegs. Kolloquien 66, München 2009, 1-19, jeweils mit Bezugnahme auf die ältere Literatur.

6 In seiner Würdigung der Arbeit Lagardes geht Rahlfs auch auf die Gründe des Scheiterns der großangelegten Pläne ein, die vorhatten, die Edition der Septuaginta in der Lebenszeit eines Forschers zu vollenden: neben der Unterschätzung der Größe des Projekts nennt Rahlfs u.a. die Arbeitsweise Lagardes, die ihn neben der Arbeit am Bibeltext stets noch habe Seitenlinien verfolgen lassen (damit kritisiert Rahlfs sicher auch Lagardes zahlreiche Editionen koptischer Texte). Vgl. Alfred Rahlfs, Paul de Lagardes wissenschaftliches Lebenswerk, im Rahmen einer Geschichte seines Lebens. Mitteilungen aus dem Septuaginta-Unternehmen, Göttingen 1928, 5883, bes. die Zusammenfassung auf S. 84.

7 Zusammenfassend in: „Paul de Lagarde und die ,Aegyptische Alterthumskunde und Koptische Sprache in Göttingen“, in: Gerald Moers, Heike Behlmer, Katja Demuß, Kai Widmaier (Hgg.), jn.t $\underline{\text { dr.w }}$ - Festschrift für Friedrich Junge, Göttingen 2006, 89-107.

8 Aegyptiaca Pauli de Lagarde studio et sumptibus edita, Göttingen 1883, IV. Andernorts spricht er von der „höchst geistreichen und tiefsinnigen ägyptischen Sprache“: Lagarde, Mittheilungen, 4 Bde, Göttingen 1884-1891, I, $178 \mathrm{f}$ (gemeint ist ebenfalls das Koptische).

9 Zu Moritz Gotthilf Schwartze s. Erika Endesfelder, Die Ägyptologie an der Berliner Universität. Zur Geschichte eines Fachgebietes, Berlin 1988, 7-9; Johannes Irmscher, „Berlin und die Koptologie“, in: Manfred Görg (Hg.), Religion im Erbe Ägyptens. Beiträge zur spätantiken Re- 
sche neutestamentliche Texte heraus, um ein Projekt seines verstorbenen Lehrers fortzusetzen und seiner Neigung zum Koptischen nachzugehen. Hier kam es zu einer ersten wissenschaftlichen Begegnung, oder besser gesagt, zu einem massiven Zusammenstoß mit dem gleichaltrigen Heinrich Brugsch, der Lagardes Jugendwerke sehr negativ rezensierte. Lagarde war von Brugsch' vielfach nicht gerechtfertigter Kritik schwer getroffen und verkaufte die Restauflage seiner koptologischen Werke als Altpapier. Die Kritik sollte ihn jahrzehntelang beschäftigen, und noch 1880 suchte er sie auf 40 Seiten zu widerlegen ${ }^{10}$. Die frühe Feindschaft zwischen Lagarde und Brugsch ist dann auch einer der Gründe, der mich dazu bewogen hat, die „Freundschaft" zwischen beiden Fächern als „nicht immer" wunderbar zu bezeichnen.

Über die persönlichen Beziehungen Brugsch' und Lagardes während ihrer gemeinsamen Göttinger Zeit ist wenig bekannt. In der Korrespondenz Lagardes in der Handschriftenabteilung der Göttinger Bibliothek gibt es nur vier Briefe von Brugsch ${ }^{11}$, der Ton ist kollegial, aber nicht freundschaftlich. Während Brugsch 1877 Göttingen verließ, blieb Paul de Lagarde und arbeitete bis zu seinem Tod 1891 an der Septuaginta-Edition. Ich habe an einem anderen Ort dargelegt, wie er immer wieder zu seiner großen Liebe, der Edition koptischer Texte zurückgekehrt ist, unter Hinantstellung seiner Arbeit am Alten Testament ${ }^{12}$.

Der Zusammenstoß zwischen Brugsch und Lagarde war jedoch nicht nur ein persönlicher, sondern eine Konfrontation zwischen zwei Wissenschaftsauffassungen, die nachhaltige Folgen auf die Geschichte der Göttinger Ägyptologie und das Fach insgesamt haben sollte.

Wir stehen in den 80er Jahren des 19. Jahrhunderts an einem Wendepunkt der Ägyptologiegeschichte: Die Ägyptologie des noch vom romantischen Interesse geprägten Heinrich Brugsch speist sich aus einer enzyklopä-

ligionsgeschichte zu Ehren von Alexander Böhlig. Ägypten und Altes Testament 14, Wiesbaden 1988, 84f; Hans Jakob Polotsky, „Egyptology, Coptic Studies and the Egyptian Language“, in: J. D. Ray (Hg.), Lingua Sapientissima. A seminar in honour of H. J. Polotsky organised by the Fitzwilliam Museum, Cambridge and the Faculty of Oriental Studies in 1984, Cambridge 1987, [5-21] 10-13.

10 Aus dem Deutschen Gelehrtenleben, Göttingen 1880, 25-65.

11 Niedersächsische Staats- und Universitätsbibliothek Göttingen, Abteilung „Handschriften und Seltene Drucke" Cod. Ms. Lagarde 150 : 170. Sie sind im Abstand von jeweils mehreren Jahren zwischen den Jahren 1857 und 1877 geschrieben worden und handeln vorwiegend vom Zugang zu Handschriften und deren Inhalten. Zusätzlich zu den Briefen Brugsch' gibt es zwei Abschriften von Briefen Lagardes (wie in seinem Briefwechsel üblich von Anna de Lagarde angefertigt).

12 „Paul de Lagarde und die ,Aegyptische Alterthumskunde und Koptische Sprache in Göttingen“ (wie Anm. 7). S. a. Polotsky, „Egyptology, Coptic Studies and the Egyptian Language“ (wie Anm. 9), 14. 
dischen Denkmälerkenntnis, die bei ihm mit einer eher intuitiv zu nennenden Übersetzungspraxis einhergeht. Die Entzifferung der Sprache ist erst in einer rezenten Vergangenheit erfolgt: bei Karrierebeginn Heinrich Brugsch' waren es gerade einmal 30 Jahre. Das Forschungsinteresse liegt in der Publikation möglichst großer Mengen der jetzt endlich lesbaren Denkmäler und ihrer Ausschöpfung für eine neue Geschichtsschreibung des Altertums, des alten Ägypten und der biblischen Geschichte. Es ist eine Pionierzeit, und Heinrich Brugsch, der Protégé Alexander von Humboldts, der schon mit 16 eine Kursivhieroglyphenschrift, das sog. Demotische, entzifferte, ist einer ihrer Helden ${ }^{13}$. Für die koptische Sprache, die wesentlich zur Entzifferung der Hieroglyphen beigetragen hat, hat dieser Held kaum mehr Verwendung.

Auf die Ausdifferenzierung der Ägyptologie aus den Altertumswissenschaften ab der Mitte des 19. Jahrhunderts sollte auch bald eine Professionalisierung, vor allem eine Philologisierung des Faches folgen. Der Held dieser neuen Generation ist Adolf Erman. Erman, ab 1883 Professor in Berlin und Gründer der sog. Berliner Schule der ägyptologischen Sprachwissenschaft ${ }^{14}$, ist der wohl nachhaltig einflußreichste Ägyptologe der Fachgeschichte in Deutschland. Unter Erman und seinen Schülern wird die Ägyptologie von einer Denkmälerwissenschaft zu einer an der semitischen Philologie orientierten textgestützten Wissenschaft. So ist es zwar wenig bekannt, aber nicht verwunderlich, daß Ermans Mentor nicht Heinrich Brugsch werden sollte, sondern der Orientalische Philologe Paul de Lagarde. Lagardes strikt philologischer Zugang zur ägyptisch-koptischen Sprache und ihren Zeugnissen findet die Bewunderung Ermans, und die beiden verbindet auch eine persönliche Freundschaft ${ }^{15}$. Erman schickte ihm seinen Studenten Georg Steindorff, der 1884 in Göttingen mit ei-

13 Die Divergenz zwischen dem „vom romantischen Impetus gegenüber der Geschichte“ geprägten Heinrich Brugsch und dem Positivismus Ermans und seiner Schule unterstreicht u.a. Sergei Stadnikow, „Die Bedeutung des Alten Orients für deutsches Denken: Skizzen aus dem Zeitraum 1871-1945“ (Print on Demand durch Propylaeum - Virtuelle Fachbibliothek Altertumswissenschaften: http://www.epubli.de/oai/propylaeumdok.uni-heidelberg.de/40), 8; s.a. U. Köhler, Göttinger Miszellen 12 (1974) (wie Anm. 4), 37-41, die zu Recht darauf hinweist, daß die unterschiedlichen Prägungen Brugsch' und Ermans auch von ihrer emotionaler Beziehung zu ihrem Forschungsgegenstand - Verehrung des Altehrwürdigen und naive Freude an der Geschichte versus emotionsfreiem Forschungsinteresse - widergespiegelt werden.

14 Wolfgang Schenkel, „Bruch und Aufbruch. Adolf Erman und die Geschichte der Ägyptologie", in Bernd-Ulrich Schipper (Hg.), Ägyptologie als Wissenschaft: Adolf Erman (1854-1937) in seiner Zeit, Berlin 2006, 224-247. S. jetzt die umfassende Darstellung von Thomas L. Gertzen: École de Berlin und "Goldenes Zeitalter" (1882-1914) der Ägyptologie als Wissenschaft. Das Lehrer-Schüler-Verhältnis von Ebers, Erman und Sethe, Berlin 2013.

15 Heike Behlmer, „Adolf Erman und Paul de Lagarde“, in: Bernd U. Schipper (Hg.), Ägyptologie als Wissenschaft: Adolf Erman (1854-1937) in seiner Zeit, Berlin 2006, 276-292. 
ner koptologischen Dissertation promoviert wurde und später als Professor in Leipzig (1904-1932) die weitere Geschichte der deutschen Ägyptologie prägen sollte ${ }^{16}$.

Der philologische Zugang, den Erman und seine Schule als die wissenschaftliche Zugangsweise zum Fach ansahen, hatte im Gegensatz zu Brugsch sehr wohl Verwendung für die koptische Sprache als jüngste Stufe des Ägyptischen und für koptologische Philologen wie Lagarde. Dies dokumentiert die Korrespondenz zwischen Erman und Lagarde eindrucksvoll ${ }^{17}$. Wofür er keine Verwendung hatte, waren die Inhalte der in koptischer Sprache geschriebenen Texte und die materielle Kultur der ägyptischen Christen. Damit verweigert die neue philologisch orientierte Ägyptologie nicht der Koptologie, aber einem großen Teil der koptischen Kultur die Freundschaft. Was für Folgen hat nun diese Verweigerung in Göttingen, wo es das zusätzliche Problem der lauwarmen Freundschaft zwischen Brugsch und Lagarde gab?

Die institutionellen Folgen der Abneigung Lagardes gegen Brugsch und die Ägyptologen seiner eigenen Generation - vor der Begegnung mit den viel jüngeren Erman und Steindorff - hätten beinahe das frühe Ende der Ägyptologie in Göttingen herbeigeführt. Lagarde schreibt 1885, daß die „Goettinger Professur der Aegyptologie ausdrücklich eingezogen sei, und daß ich selbst es gewesen [bin], der in der Fakultät ... den Antrag sie durch eine dritte Professur der klassischen Philologie zu ersetzen gestellt $[\text { hat }]^{\text {"18 }}$. Erst durch die Ernennung des späteren Bibliotheksdirektors Richard Pietschmann zum a.o. Professor für Ägyptologie und altorientalische Geschichte im Jahre 1889 wird die Ausbildung im Fach wieder

16 Steindorffs Dissertation hatte den Titel „Prolegomena zu einer koptischen Nominalclasse“. Auch seine Berliner Habilitationsschrift „Koptische Nominalbildung“ (1890) hatte die koptische Sprache zum Gegenstand, und noch aus seinem kalifornischen Exil, in das er sich noch 1939 vor den Verfolgungen des Nationalsozialismus hatte retten können, verfaßte er eine koptische Grammatik. Steindorffs Wirken und Bedeutung für die Ägyptologie werden ausführlich gewürdigt von Elke Blumenthal, Altes Ägypten in Leipzig. Zur Geschichte des Ägyptischen Museums und des Ägyptologischen Instituts an der Universität Leipzig, Leipzig 1981, 15-31. Zu Steindorffs Schicksal während des Nationalsozialismus und des 2. Weltkriegs vgl. zuletzt Thomas Schneider, „Ägyptologen im Dritten Reich. Biografische Notizen anhand der sogenannten ,Steindorff-Liste“ “, Journal of Egyptian History 5 (2012), [120-247] 127-135.

17 Heike Behlmer, „Ein neo-koptischer Brief Adolf Ermans an Paul de Lagarde - Zeugnis für eine wissenschaftsgeschichtliche Wende in der Erforschung des Koptischen“, Lingua Aegyptia 11 (2003), 1-12.

18 Brief vom 6.5.1885 an Minister von Goßler (Niedersächsische Staats- und Universitätsbibliothek Göttingen, Abteilung „Handschriften und Seltene Drucke“ Cod. Ms. Lagarde 150 : 438). 
aufgenommen. Nach dem Weggang Pietschmanns kam 1900 ein anderer Erman-Schüler, Kurt Sethe, nach Göttingen ${ }^{19}$.

Die Ägyptologie wurde durch Pietschmann und Sethe gerettet, aber die Freundschaft nur zum Teil, und damit komme ich zu den Auswirkungen auf die Fachentwicklung. Unter Sethes enormem Publikationsausstoß gibt es in der Nachfolge seines Lehrers Erman eine ganze Reihe von grundlegenden Beiträgen zur koptischen Philologie und Sprachwissenschaft ${ }^{20}$. Sethe erklärte jedoch öffentlich, daß sein Fach nur an der koptischen Sprache interessiert sei. Die gesamte koptische Literatur wolle er den Theologen überlassen, da ihr Inhalt vorwiegend religiöser Natur sei ${ }^{21}$. Sethes eigener Nachfolger Hermann Kees war ein Spezialist für ägyptische Religionswissenschaft. Die koptische Sprache war aber weiterhin ein integraler Bestandteil ägyptologischer Lehre. Wie seine nachgelassenen Skripte in unserem Archiv zeigen, las Kees u.a. koptische Bibeltexte mit den Studierenden ${ }^{22}$.

Diese Göttinger Beobachtungen lassen sich für die Zeit bis nach dem 2. Weltkrieg verallgemeinern. Im Gegensatz zur Ägyptologie bildete die Koptologie kein eigenes Fach heraus. Stattdessen wurden die koptischen Texte als Appendix der Ägyptologie betrachtet und ohne viel Interesse an den Inhalten und textual communities, die sie produziert hatten, gelesen und sprachgeschichtlich analysiert. Die Beschäftigung mit den Inhalten des „dürren Spätling $[s]$ der altberühmten Nilkultur“, so der Ägyptologe Siegfried Morenz 195223, wurde an die Theologie ausgelagert.

Dennoch sind dieser, nennen wir es „selektiven“ Freundschaft, immer wieder exzellente Koptologen und ägyptologisch-koptologische Sprachwissenschaftler entsprungen. So sollte Hans Jakob Polotsky, ein Schüler Kees' und Alfred Rahlfs', der 1934 Deutschland verlassen mußte, zum Begründer der modernen ägyptisch-koptischen Linguistik werden. Auch in der jünge-

19 Jürgen Horn, „Daten zur Geschichte der Ägyptologie in Göttingen“, Göttinger Miszellen 28 (1978), 11-19, gibt eine hilfreiche Übersicht der Göttinger Ägyptologiegeschichte. S. dort S. 12 auch zu Pietschmanns Rückkehr nach Göttingen im Jahre 1903 mit weiteren Lehrverpflichtungen in der Ägyptologie.

20 Neben den sprachgeschichtlichen Untersuchungen Das aegyptische Verbum im altägyptischen, neuaegyptischen und koptischen, Leipzig 1899-1902 und Der Nominalsatz im Ägyptischen und Koptischen, Leipzig 1916 hat Sethe weit mehr als 20 Aufsätze zu Einzelfragen der ägyptischkoptischen Sprache hinterlassen.

21 Diese Aussage Sethes von 1921 wird zitiert von Johannes Irmscher, „Berlin und die Koptologie“, in: Manfred Görg (Hg.), Religion im Erbe Ägyptens, Beiträge zur spätantiken Religionsgeschichte zu Ehren von Alexander Böhlig. Ägypten und Altes Testament 14, Wiesbaden 1988, [83-93] 83 .

22 Auch nach Ausweis der Vorlesungsverzeichnisse gibt Hermann Kees seit 1928 regelmäßig koptische Sprachkurse.

23 Siegfried Morenz, „Die koptische Literatur“", in: Handbuch der Orientalistik, 1. Bd., 2. Abschn., Leiden 1952, 219. 
ren Vergangenheit hat sich die von Lagarde und Erman begründete Verbindung von ägyptologischer und koptologischer Philologie und Sprachgeschichte in Göttingen fortgesetzt. Besonders eindrucksvoll geschah dies unter der Ägide von Wolfhart Westendorf, einem Mitglied in der vierten Generation der von Erman begründeten Berliner Schule der ägyptologischen Sprachwissenschaft. Sein sprachgeschichtlich orientiertes Koptisches Handwörterbuch ist zu einem Standardwerk geworden ${ }^{24}$. Die Arbeiten Friedrich Junges und Antonio Loprienos zur ägyptisch-koptischen Sprachgeschichte haben diese Tradition fortgeschrieben ${ }^{25}$. Damit ist die Freundschaft der Ägyptologie und Koptologie trotz aller nicht so wunderbaren Momente auf dem Gebiet der Sprachgeschichte seit fast 150 Jahren von Lagarde bis zu Westendorf und seinen Schülern fast unverändert wunderbar geblieben.

Die Probleme dieser selektiven Freundschaft lagen auf anderen Gebieten. Durch das mangelnde Interesse an der Literatur und der religiösen und materiellen Kultur Ägyptens nach den Pharaonen ist die Geschichte der Koptologie vom 19. Jh. bis nach dem 2. Weltkrieg eine Geschichte von Versäumnissen. Weil die jüngeren archäologischen Schichten in den Pionierzeiten der ägyptischen Archäologie in der 2. Hälfte des 19. Jahrhunderts als unwichtiger Lehmschutt angesehen wurden, der die imposanten Steinbauten der Pharaonen verschandelte, wurden Siedlungen, Klöster und Friedhöfe vielfach ignoriert, zerstört und ohne Dokumentation und Publikation abgeräumt. Weil das Koptische als Anhängsel des pharaonischen Ägyptischen angesehen wurde, fehlt noch heute eine Aufnahme des Gesamtwortschatzes der Sprache. Es gibt keinen Thesaurus Linguae Copticae und kein vollständiges Wörterbuch der koptischen Sprache. Weil die Literatur des ägyptischen Christentums in koptischer Sprache auch von den Theologen, an die sie ausgelagert worden war, oft als nicht originell oder wertvoll angesehen wurde, sind wenige koptische Texte so publiziert, daß es modernen Ansprüchen genügen würde.

Von der Theologie und von der Ägyptologie für Jahrzehnte fast verlassen, erfuhr die Koptologie nach dem 2. Weltkrieg eine Renaissance, dann auch als akademisches Fach. Dies war zum einen der Expansion der Universitäten geschuldet, zum anderen dem erneuerten Interesse am Gegenstand des Faches, das durch sensationelle neue Funde, wie die auf Koptisch überlieferten gnostischen oder manichäischen Schriften, verstärkt wurde. In der

24 Wolfhart Westendorf, Koptisches Handwörterbuch, Heidelberg 1977.

25 Um nur die wichtigsten monographischen Studien zu nennen: Friedrich Junge, Neuägyptisch. Einführung in die Grammatik, 3. verb. Aufl., Wiesbaden 2008; Antonio Loprieno, Ancient Egyptian. A Linguistic Introduction, Cambridge 1995. 
Expansionsphase der Universitäten gelang es 1971 in Münster, die Koptologie als Professur institutionell zu verankern. Dies geschah in Göttingen trotz aller Bemühungen Wolfhart Westendorfs in dieser Form nicht, aber das Fach wurde seit dem Ende der 60er Jahren in Forschung und Lehre durch seine Mitarbeiter Jürgen Horn und Christa Müller vertreten. Heute ist die akademische Lage der Koptologie uneinheitlich. Das Interesse wächst weiter, inzwischen auch unter den koptisch-orthodoxen Christen, von denen inzwischen Hunderttausende in der Diaspora in Europa, Amerika und Australien leben. Mancherorts fallen Stellen Sparmaßnahmen zum Opfer, in den Einwanderungsländern und in Ägypten dagegen bemüht man sich um Stiftungslehrstühle ${ }^{26}$.

In der Forschung versucht die Koptologie, die Versäumnisse nachzuholen, die aus der nicht immer wunderbaren Freundschaft mit der Ägyptologie entstanden sind. In Münster arbeitet Stephen Emmel mit einem internationalen Team seit zehn Jahren an der Gesamtedition des wohl bedeutenden Autors in koptischer Sprache, des Abtes Schenute ${ }^{27}$. In Leipzig wird ein Datenbankprojekt zu den Lehnwörtern im Koptischen die Lexikographie der Sprache auf eine neue Basis stellen ${ }^{28}$. Zu beiden Forschungsgebieten habe ich ein klein wenig beigetragen ${ }^{29}$. In meiner weiteren wissenschaftlichen Tätigkeit möchte ich vor allem in zwei Richtungen arbeiten. Zum einen möchte ich weiter der Wissenschaftsgeschichte unseres Faches nachspüren und u.a. die ägyptologische Korrespondenz Paul de Lagardes herausgeben ${ }^{30}$. Insbesondere aber möchte ich mich ebenso wie

26 Durch private Spenden unterstützte Programme in Koptologie gibt es an der American University in Cairo (www.aucegypt.edu/huss/sape/egpt/), der Claremont Graduate University (www.cgu.edu/pages/9884.asp), der Macquarie University Sydney (www.anchist.mq.edu.au) und der University of Toronto (www.utoronto.ca/nmc; alle Webseiten zuletzt besucht am 30.6.2012),

27 Stephen Emmel, Shenoute's Literary Corpus. 2 Bde. Corpus Scriptorum Christianorum Orientalium 599-600 (Subsidia 111-112). Leuven 2004.

28 http://www.uni-leipzig.de/ ddglc/ (zuletzt besucht am 30.6.2012).

29 Zu Schenute und seinem Kloster u.a.: „The City as Metaphor in the Works of Two Panopolitans: Shenoute and Besa“, in: A. Egberts, B. P. Muhs and J. van der Vliet (Hgg.), Perspectives on Panopolis. An Egyptian Town from Alexander the Great to the Arab Conquest. Acts from an International Symposium held in Leiden on 16, 17 and 18 December 1998. Papyrologica LugdunoBatava 31, Leiden-Boston-Köln 2002, 13-27; „,Our Disobedience Will Punish Us...': The Use of Authoritative Quotations in the Writings of Besa“, in: Dieter Kessler et al., (Hgg.), TexteTheben-Tonfragmente. Festschrift für Günter Burkard, Wiesbaden 2009, 37-54; „The Use of the Psalms in Shenoute's Tractate He Who Sits Upon His Throne" (im Druck in einer Festschrift); zur Lehnwortforschung: „Differentiating lexical borrowing according to semantic fields and text types - a case study“ (im Druck in den Akten der Konferenz „Linguistic Borrowings into Coptic“, Leipzig 26.-28. April 2010, hg. von Sebastian Richter und Eitan Grossman).

30 Es geht um eine ausbaufähige elektronische Edition, die einen wichtigen Teil der ägyptologischen Netzwerke der Zeit von 1860-1890 sichtbar machen und einen Schwerpunkt auf der 
der genannten Projekte der lange vernachlässigten koptologischen Grundlagenforschung widmen, idealerweise einem weiteren wichtigen Desiderat der Forschung, das seine Wurzeln ebenfalls in der Zeit Lagardes hat: einer digitalen Edition des koptischen Alten Testaments. Die Bibel ist die Grundlage fast der gesamten koptischen Literatur und religiösen Kultur, und der Gewinn für das Fach würde enorm sein. Die durch ein solches Projekt gewonnenen digitalen Texte würden zweierlei tun: die ägyptologischkoptologische Sprachwissenschaft befördern und u.a. in einen zukünftigen Thesaurus Linguae Copticae einfließen und die Erforschung der koptischen Literatur und religiösen Kultur voranbringen. Damit würde zum einen die Traditionslinie der Freundschaft zwischen ägyptischer und koptischer Philologie und Linguistik in Göttingen fortgesetzt werden können, die ich hier nachzuzeichnen versucht habe. Gleichzeitig aber würden zu einem kleinen Teil die Nachteile für die Koptologie ausgeglichen werden können, die aus der Selektivität dieser Freundschaft, d.h. dem mangelnden Interesse der Ägyptologie an den Inhalten der Texte und ihren Verfassern, entstanden sind. Ich weiß noch nicht, ob diese Pläne sich verwirklichen lassen, aber wenn ja, so wäre dies für mich ein wunderbarer Beitrag zur Freundschaft zwischen beiden Fächern.

orientalistisch-ägyptologischen Praxis Lagardes und seiner Zeitgenossen haben soll. Sie wird damit einen Schritt hin zu der von Bernhard Neuschäfer eingeforderten Gelehrtenbiographie Lagardes darstellen: „Ulrich Sieg: Deutschlands Prophet. Paul de Lagarde und die Ursprünge des modernen Antisemitismus", in: Göttingische Gelehrte Anzeigen 261 (2009), 91-112. 